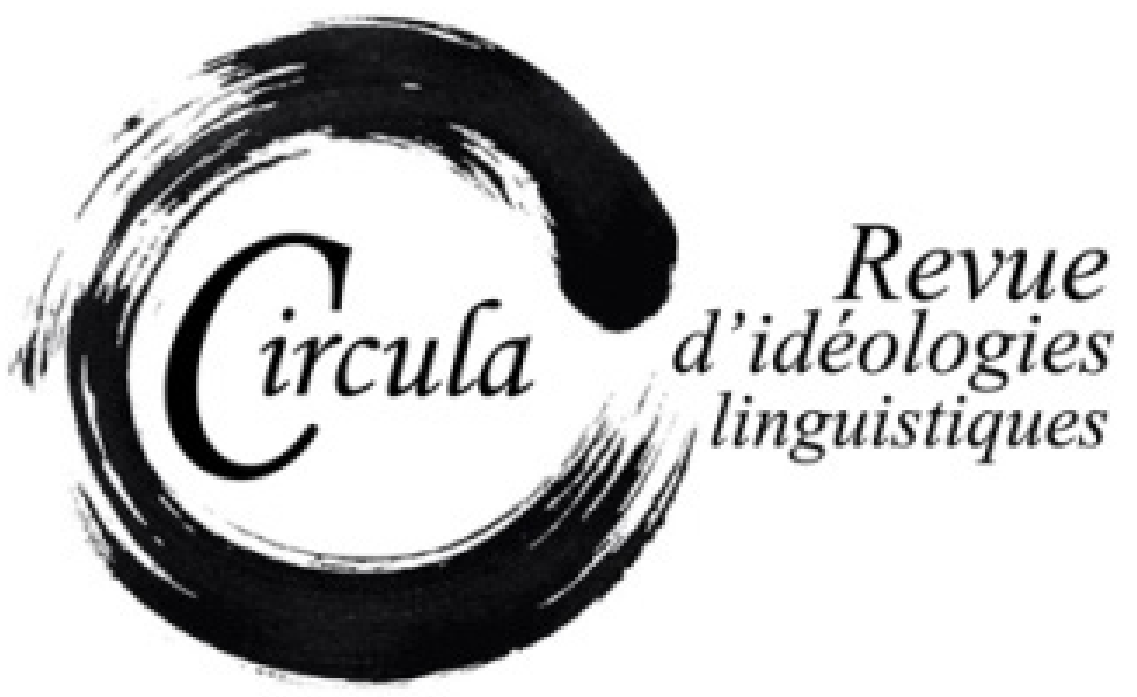

TITRE: 40 ANS APRÈS, QU'EN EST-IL DE LA LOI 101 ? REPRÉSENTATIONS ET DISCOURS CONFLICTUELS DANS LA PRESSE QUÉBÉCOISE

Auteur(s): GenevièVe Bernard Barbeau, Université du Québec À Trois-Rivières

Revue: CirCula, NUMÉRO 7

PAGES: $51-69$

ISSN: 2369-6761

DIRECTEUR: WIM REMYSEN

URI: HTTP://HDL.HANDLE.NET/11143/14494

DOI: HTTPS://DOI.ORG/10.17118/11143/14494 


\section{0 ans après, qu'en est-il de la loi 101 ? Représentations et discours conflictuels dans la presse québécoise}

Geneviève Bernard Barbeau, Université du Québec à Trois-Rivières genevieve.bernardbarbeau@uqtr.ca

Résumé : S'il est indéniable que la Charte de la langue française (loi 101) a joué un rôle fondamental dans l'histoire du Québec, elle fait encore aujourd'hui l'objet de nombreuses controverses. Entrée en vigueur en août 1977, la loi 101 a récemment fêté ses 40 ans, ce qui n'est pas passé sous silence dans les médias. Cet article porte sur les représentations qui entourent la loi 101 et qui ont été véhiculées à l'occasion de cet anniversaire dans la presse écrite québécoise francophone et anglophone. L'analyse de ce corpus, provenant de médias qui s'adressent à des communautés linguistiques différentes et à des lecteurs partageant des points de vue politiques divergents, permettra de faire ressortir non seulement les différents regards portés sur la législation linguistique québécoise à l'heure actuelle, mais également la façon dont ces points de vue antagonistes sont hiérarchisés les uns par rapport aux autres.

Mots-clés : loi 101 ; discours épidictique ; débat; médias; Québec

Abstract: While it is undeniable that the French Language Charter (Bill 101) has played a fundamental role in the history of Quebec, it is still the subject of much controversy today. Passed into law in August 1977, Bill 101 recently celebrated its 40th anniversary, which did not go unnoticed by the media. This article focuses on the portrayals of Bill 101 by Quebec's print media, both in French and English, on the occasion of this anniversary. The analysis of this data collection, which comes from newspapers of different linguistic communities and readers sharing divergent political views, will highlight the various perspectives on Quebec's current language legislation.

Key words: Bill 101; epideictic rhetoric; debate; media; Quebec 


\section{Introduction}

La Charte de la langue française, aussi connue sous l'appellation loi 101, a joué un rôle fondamental dans l'évolution du Québec en ce qu'elle a transformé les rapports de force entre les groupes linguistiques et qu'elle a grandement participé à l'émancipation des Québécois francophones. Entrée en vigueur le 26 août 1977, la loi 101 a récemment célébré ses 40 ans. Cet anniversaire n'est pas passé sous silence dans les médias québécois, ni dans la communauté scientifique d'ailleurs ${ }^{1}$, comme ça a également été le cas pour chacun de ses anniversaires importants $\left(10^{e}, 25^{e}, 30^{e} \text {, etc. }\right)^{2}$. Et si l'heure est alors au bilan, les travaux qui s'inscrivent dans ce cadre portent habituellement sur les effets de la loi 101 sur les communautés linguistiques (Oakes, 2004), notamment au plan démographique (Levine, 2002), et sur son rôle dans l'intégration des non-francophones à la communauté francophone (McAndrew, 2002; Sarazin, 2002).

Dans cet article, nous nous intéressons moins aux effets de la loi 101 qu'aux représentations qui l'entourent et qui ont été véhiculées dans les journaux québécois de langue française et anglaise à l'occasion de son $40^{\circ}$ anniversaire. L'analyse de ce corpus, provenant de médias qui s'adressent à des communautés linguistiques distinctes et à des lecteurs qui partagent des points de vue politiques divergents, permettra de faire ressortir les différents regards posés sur la législation linguistique québécoise à l'heure actuelle et de faire état des arguments mobilisés pour étayer ces points de vue antagonistes.

\section{Le Québec et la loi 101 : un bref rappel historique}

La loi 101 a été proclamée après des décennies de vifs débats et d'importantes tensions linguistiques au Québec qui ont mené à l'adoption de différentes lois en matière de langue (Loi pour promouvoir la langue française [loi 63, 1969] et Loi sur la langue officielle [loi 22, 1974]), qui ont été jugées insatisfaisantes et qui ont été successivement rejetées³. La loi 101 est donc adoptée alors que le conflit entre les groupes linguistiques est à son apogée. Rappelons qu'à l'époque, bien que nettement majoritaires en termes démographiques au Québec, les francophones se trouvaient en

1. Pensons notamment au colloque Les 40 ans de la loi 101 : la Charte de la langue française et les communautés québécoises d'expression anglaise, 1977-2017, organisé par Lorraine O'Donnell (Université Concordia), Patrick Donovan (Université Laval) et Dominique Pépin-Fillion (Université de Moncton), qui s'est tenu en mai 2017 dans le cadre du congrès de l'Association francophone pour le savoir (Acfas).

2. Mentionnons entre autres un numéro hors série de la Revue d'aménagement linguistique, consacré, en 2002, aux 25 ans de la loi 101 : L'aménagement linguistique au Québec : 25 ans d'application de la Charte de la langue française. Plusieurs des articles publiés dans ce numéro sont ici cités.

3. Pour une présentation détaillée de l'évolution de la législation linguistique québécoise, nous renvoyons à Corbeil (2007). Soulignons seulement, parmi les nombreuses mesures qui ont suscité ou attisé le conflit entre les groupes à la suite des premières lois, le libre choix de la langue d'enseignement, qui a été réaffirmé par la loi 63 avant d'être en partie restreint - trop pour certains, pas suffisamment pour d'autres - par la loi 22. 
situation d'infériorité politique, économique, sociale et linguistique, et que le rapport de force entre anglophones et francophones était inégal.

C'est pour pallier cette inégalité que, quelques mois après son élection, le gouvernement du Parti québécois, dirigé par René Lévesque, présente un énoncé de politique intitulé La politique québécoise de la langue française (Gouvernement du Québec, 1977), où il est proposé de faire du français la véritable langue commune de tous les Québécois en tenant compte de quatre principes : le français est plus qu'un simple outil de communication au Québec, il s'agit d'un véritable milieu de vie ; il faut respecter les groupes ethniques minoritaires, leurs langues et leurs cultures; apprendre d'autres langues que le français est bénéfique pour tous les Québécois ; le statut du français au Québec est une question de justice sociale, c'est-à-dire que les francophones doivent prendre la place qui leur revient, principalement dans le monde du travail, afin de ne plus être dominés par la minorité anglophone (Corbeil, 2007 : 231-234).

Après avoir diffusé cet énoncé de politique, le gouvernement fait adopter la loi 101 en août 1977, loi qui poursuit cinq objectifs : « Définir la nature linguistique de la société québécoise, assurer l'intégration scolaire des enfants immigrants, franciser le monde du travail, pourvoir aux conditions de respect de la majorité francophone, créer les organismes chargés de la mise en œuvre de la Charte » (Rocher, 2008 : 343). Le français est ainsi officiellement proclamé langue de la législation, de la justice, de l'administration publique, des organismes parapublics, du travail, du commerce, des affaires et de l'enseignement. En ce qui a trait à l'éducation, un des éléments centraux du conflit linguistique des années 1960-19704, la loi 101 s'inscrit en faux contre les lois 63 et 22 : de la maternelle à la fin du secondaire, l'éducation est obligatoirement reçue en français pour tous les élèves québécois, sauf pour ceux dont l'un des parents a fréquenté l'école primaire et secondaire anglaise au Québec ${ }^{5}$ et pour ceux qui fréquentaient déjà l'école de langue anglaise avant l'entrée en vigueur de la Charte, de même que pour leurs frères et sœurs. Ainsi, « les francophones sont promus tout à coup à un véritable statut de majoritaires et constatent que leur langue occupera désormais la première place dans les activités publiques fondamentales »(Bernard, 2008 : 360). Essentiellement, la loi 101 a défini la société québécoise comme une société de langue française, et les rapports entre les groupes linguistiques ont ainsi été transformés.

4. Cet élément est d'ailleurs encore au cœur des débats aujourd'hui, comme nous le montrerons dans l'analyse. C'est pourquoi nous accordons ici une importance particulière à la question de la langue d'enseignement.

5. Cet aspect de la loi 101 sera nommé la clause Québec lorsqu'il sera contesté devant les tribunaux quelques années plus tard. En 1984, la clause Québec est déclarée inopérante par la Cour suprême en vertu de la clause Canada, qui reconnaît le droit à l'enseignement dans la langue de la minorité (l'anglais dans le cas du Québec, le français dans les autres provinces) pour tous les enfants dont l'un des parents a reçu son éducation dans cette langue où que ce soit au Canada, de même que pour leurs descendants. La situation demeure toutefois inchangée pour les francophones et pour les allophones. 
Depuis sa mise en place, la loi 101 a été modifiée à plusieurs reprises, que ce soit pour prendre en considération l'évolution de la situation linguistique et les changements qui en découlent ou parce que certaines dispositions ont été invalidées, à la suite de contestations juridiques, car jugées incompatibles avec, entre autres, la Constitution canadienne ou avec la Charte québécoise des droits et libertés de la personne (Woehrling, 2005). Malgré ces modifications, la loi 101 constitue encore aujourd'hui le fondement de la politique linguistique québécoise et plusieurs la considèrent comme une des lois phares du Québec (Oakes et Warren, 2009). Mais de nombreuses critiques ont aussi été adressées à la loi 101 et continuent de l'être encore aujourd'hui. C'est cette ambiguïté - entre regard positif et négatif porté sur la loi 10140 ans après son adoption - que nous souhaitons ici mettre de l'avant.

\section{Cadre théorique et méthodologique}

\subsection{Corpus d'analyse}

Notre analyse est fondée sur un corpus constitué de quatre des principaux journaux québécois, tant francophones (Le Devoir, Le Journal de Montréal et La Presse) qu'anglophone (The Gazette). À partir d'une recherche menée au moyen des mots-clés loi 101 et Charte de la langue française dans la base de données Eureka, nous avons recueilli l'ensemble des textes qui ont été publiés sur le sujet entre janvier et septembre 2017 dans les trois journaux francophones. Nous avons ensuite épluché la version électronique du journal The Gazette, qui n'est pas inclus dans cette base de données, pour établir un corpus comparable. De l'ensemble de ces textes, nous avons sélectionné ceux qui portaient spécifiquement sur les 40 ans de la loi 101, et non sur la politique linguistique québécoise de façon générale, et qui faisaient une large part à l'opinion, qu'il s'agisse d'éditoriaux, de chroniques ou de lettres d'opinion de spécialistes ou de lecteurs. Au total, le corpus comprend 54 textes, 38 en français (22 au Devoir, 5 à La Presse et 11 au Journal de Montréal) et 16 en anglais (tous à The Gazette). La majorité ont été publiés autour du 26 août, date anniversaire de la loi, mais certains ont été rédigés dans les semaines, voire dans les mois, qui précèdent, signe de l'importance accordée à l'événement dans l'espace médiatique québécois.

L'autorité qui est conférée aux auteurs de ces différents textes est variable, mais la prise en considération du discours de chacun est pertinente pour faire le point sur la façon dont est envisagée la loi 101 en 2017. Les spécialistes (politiciens, chercheurs, individus ayant participé à l'élaboration de la politique linguistique québécoise, etc.) donnent à voir le discours de ceux qui ont façonné le paysage politique et linguistique québécois ou encore qui en ont fait l'objet de nombreux travaux de recherche. Leur opinion sur la loi 101 est donc souvent fondée sur une expérience de premier plan. Le discours des journalistes, quant à lui, est pris en considération parce que c'est notamment à partir de lui que s'informe une tranche importante de la population, ici à propos des questions linguistiques qui animent le Québec, et que les journaux constituent en ce sens un lieu propice à la circulation 
des représentations et des idéologies linguistiques (voir notamment Fowler, 1991) ${ }^{6}$. Enfin, le discours des citoyens s'inscrit certes dans une autre perspective parce qu'il ne constitue pas à proprement parler un discours d'autorité, contrairement à celui des spécialistes et des journalistes, mais il est néanmoins révélateur des représentations qui circulent dans la communauté et qui participent à construire la perception actuelle de la loi 101, qui se donne aussi à voir et à entendre dans l'espace public. Ainsi, l'analyse de textes provenant de sources variées et rédigés par différents auteurs permet d'envisager un ensemble de discours qui, s'ils ne se nourrissent pas nécessairement l'un l'autre, nourrissent assurément le discours des Québécois en matière de langue et de politique linguistique et, partant, contribuent au bilan médiatique qui est fait des 40 ans de la loi 101.

\subsection{Bilan anniversaire et discours épidictique}

Nous avons mené notre analyse suivant les travaux d'Amossy $(2010,2014)$ sur l'argumentation et sur le discours polémique. En raison de leur objet, ces travaux fournissent un cadre approprié pour interpréter un corpus de discours faisant état de dissensions où chaque auteur donne à voir son opinion sur une loi qui a fait l'objet de nombreuses controverses. Mais plus spécifiquement, nous nous intéressons au croisement entre ces dissensions (tensions identitaires et linguistiques, rapports conflictuels de longue date entre les groupes, etc.) et la façon de les aborder dans un contexte bien précis, le bilan d'une loi autant applaudie que décriée. C'est ainsi que nous associons ce contexte à un genre médiatique que l'on pourrait qualifier de bilan anniversaire.

Par définition, le bilan anniversaire est fondé sur le rappel de faits marquants qui s'échelonnent sur une période circonscrite. Il appartient au genre épidictique, pour reprendre la triade d'Aristote, à côté des genres délibératif et judiciaire. Sa fonction est de louer et de blâmer, comme le font aussi, par exemple, les portraits de personnalités publiques ou les éloges funèbres (Dominicy et Frédéric, 2001). Le bilan anniversaire, comme tout discours épidictique, «se propose d'accroître l'intensité de l'adhésion à certaines valeurs » (Perelman et Olbrechts-Tyteca, 1988:67), ce qui lui confère non seulement une dimension argumentative, mais également une fonction sociale s'apparentant à un rituel où les membres d'une communauté se rassemblent pour partager leurs souvenirs d'une personne ou d'un événement (Pernot, 1993). Mais à l'exception du rappel de faits tragiques - guerres, génocides, etc. -, il participe aussi à un jeu rhétorique qui consiste à relever des points positifs et négatifs, des bons et des moins bons coups, et ce, peu importe l'opinion défendue par l'énonciateur. Cela ne signifie pas pour autant que le discours est livré tout en nuances de manière à atteindre - ou à faire atteindre - un consensus. Au contraire, malgré ce jeu rhétorique, des prises de position nettes peuvent émerger. Et de tous les précédés et structures qui contribuent à l'articulation des discours, certains sont plus adéquats que d'autres pour rendre manifestes ces prises de position. Une première lecture exploratoire des 54 articles constituant notre corpus nous a permis de repérer la récurrence d'une de ces structures, qui articulera notre analyse : la concession.

6. En témoignent aussi les nombreux travaux publiés ici même dans la revue Circula. 


\subsection{La concession : une structure révélatrice de tensions argumentatives}

La concession est un procédé rhétorique qui exprime une tension entre deux thèses opposées. Morel (1996 : 5) la définit comme « un mouvement argumentatif en deux temps [où l']orateur commence par reconnaître la validité d'un argument qu'il prête à son adversaire dans le débat. Puis il énonce à la suite un contre-argument qui vient en restreindre la portée ou le détruire ». La concession contient deux éléments explicites : un énoncé concédé, qui est «l'anticipation d'une objection à une déclaration » (Vincent et Heisler, 1999 : 24), et un contre-argument, qui « a pour but de l'éliminer (soit d'en minimiser l'importance, soit de l'invalider, tout en maintenant sa valeur de vérité) » (Vincent et Heisler, 1999 : 25) et qui est généralement introduit par un marqueur de concession (mais, or, cependant, etc.). Elle contient également une conclusion, qui peut être énoncée explicitement ou inférée à partir du contre-argument, avec lequel elle est co-orientée. La concession prototypique se présente sous la forme « $X$ mais $Y$ donc $Z$ », où X est l'énoncé concédé, $Y$ est le contre-argument et $Z$, la conclusion qui en découle. L'exemple suivant, qui provient de notre corpus d'analyse, permet d'en illustrer plus concrètement le fonctionnement:

(1) On peut comprendre les parents francophones de souhaiter un meilleur apprentissage de l'anglais pour leurs enfants, mais il faut être inconscients pour ne pas voir le danger d'un tel retour au passé. (David, Le Devoir, 26 août 2017)

L'énoncé concédé (« on peut comprendre les parents francophones de souhaiter un meilleur apprentissage de l'anglais pour leurs enfants ») est suivi d'un contre-argument (" mais il faut être inconscients pour ne pas voir le danger d'un tel retour au passé »), à partir duquel il est possible d'inférer la conclusion suivante : il ne faut pas assouplir la loi 101.

Cette hiérarchisation entre énoncé concédé et contre-argument peut aussi se faire dans la macrostructure du texte, c'est-à-dire quand son ensemble est considéré, et non uniquement au sein d'un même énoncé. C'est le cas de plusieurs articles du corpus, dont l'un d'entre eux peut être schématisé ainsi : énoncés concédés (onze paragraphes servant à rappeler les avancées permises par la loi 101) suivis de contre-arguments (cinq paragraphes portant sur la situation actuelle, jugée problématique, et introduits par le marqueur et pourtant), qui mènent à une conclusion explicite : « Bref, quand il s'agit du statut et de l'avenir de la langue française au Québec, si l'on ne doit pas être pessimiste, il est incongru d'être optimiste ${ }^{7}$ (Rocher, Le Devoir, 26 août 2017).

7. La conclusion de l'article est elle-même formulée sous forme concessive, où l'énoncé concédé est «si l'on ne doit pas être pessimiste » et le contre-argument, « il est incongru d'être optimiste ». La conclusion générale du texte, qui provient de ce contre-argument et qui découle également de l'ensemble des points jugés problématiques soulevés par l'auteur, témoigne d'une attitude plutôt négative envers l'état actuel de la situation linguistique québécoise. 
La concession « permet, sans incohérence, de se faire l'avocat d'une thèse tout en apportant son assentiment à l'un des arguments qui militent en faveur de l'autre » (Danon-Boileau et Morel, 1992: 7). En d'autres termes, recourir à la concession permet d'exprimer différents messages (Krieg-Planque, 2010) et de coupler, bien qu'en les hiérarchisant, deux arguments qui, en temps normal, seraient antithétiques. Le discours est alors plus nuancé, du moins en apparence :

La concession s'oppose surtout aux dangers de la démesure : elle exprime le fait que l'on réserve un accueil favorable à certains arguments réels ou présumés de l'adversaire. En restreignant les prétentions, en abandonnant certaines thèses, en renonçant à certains arguments, l'orateur peut rendre sa position plus forte, plus aisée à défendre, et témoigner en même temps dans le débat de fair-play et d’objectivité (Perelman et Olbrechts-Tyteca, 1988 : 646).

En somme, le recours à la concession participe à la construction d'un discours orienté au plan argumentatif puisqu'en présentant leurs valeurs et leurs points de vue et en les opposant à ceux d'autrui, les individus se positionnent par rapport à un objet donné, ici la loi 101. Ainsi, la concession est à mettre en relation avec le genre épidictique, dont elle vient syntaxiquement produire l'effet d'opposition entre argument et contre-argument.

Pour chacun des articles du corpus, nous avons identifié les différentes hiérarchisations établies au moyen de structures concessives entre les points de vue donnés sur la loi 101, ce qui nous a permis de montrer les nœuds de tension autour de cette thématique dans le discours médiatique québécois. Si chacune des concessions a été analysée individuellement, ce sont surtout les conclusions générales qui en découlent qui nous intéressent. Nous partons de l'idée selon laquelle ces conclusions témoignent de l'opposition générale qui est présente dans l'ensemble du discours public. Cette diversité dans les conclusions fait état de tensions entre les différents points de vue des auteurs prenant part au bilan anniversaire de la loi 101.

\section{Analyse}

La quasi-totalité des textes analysés respectent le genre qu'est le bilan anniversaire en ce que leurs auteurs soulèvent et commentent à la fois des points jugés forts et faibles de la loi 101. Seuls trois articles font état d'un seul point de vue, amené sans référence aux différentes opinions qui circulent également dans l'espace public. La structure même de la majorité des textes appelle donc à une série d'arguments et de contre-arguments, d'éléments positifs et négatifs qui, forcément, sont hiérarchisés. 


\subsection{Regards positifs sur la loi 101}

La plupart des articles à l'étude contiennent des points positifs, et ce, peu importe le journal où ils sont publiés. Sont principalement soulignés la nécessité de la loi 101 en 1977 et le rôle qu'elle a joué dans l'émancipation des francophones, comme l'illustrent les exemples suivants, tirés de chacun des journaux du corpus:

(2) Il ne fait aucun doute que la Charte de la langue française a changé le visage du Québec et les Québécois eux-mêmes comme aucune autre loi ne l'a fait. Il y a le Québec d'avant la loi 101 et celui d'après. (David, Le Devoir, 26 août 2017)

(3) Véritable projet de société, la Charte de la langue française a ainsi changé le visage du Québec en profondeur, malgré ses reculs. Elle a renversé l'ancien ordre établi, elle a servi d'ancrage identitaire, elle a cimenté un pacte linguistique. (Cardinal, La Presse, 26 août 2017)

(4) Quarante ans après son adoption, la loi 101 doit être regardée comme un acte fondateur du Québec moderne. II s'agit d'un des gestes les plus forts d'affirmation de ce Québec qui devenait le foyer d'une majorité francophone sur son territoire, plutôt qu'une minorité dans le Canada. (Dumont, Le Journal de Montréal, 26 août 2017)

(5) History has shown that they [les partisans de la loi 101] were right on the principle of protecting French. Freedom of choice in education would have led to a catastrophic decline of French, at least in Montreal and that has been avoided. It made no sense for commercial signs to be in English only, and legislating the obligation to advertise and announce in French was both reasonable and just. (Grey, The Gazette, 24 mai 2017)

En ce sens, la loi 101 est présentée comme un important moteur de changement non seulement positif, mais nécessaire, voire comme un acte de résistance souvent envisagé avec fierté, ce qui est accentué par le vocabulaire mélioratif employé ( "véritable projet de société », « acte fondateur du Québec moderne ») ou encore par les affirmations présentées comme irréfutables (« il ne fait aucun doute que »). La législation linguistique est alors perçue comme émancipatrice pour la communauté francophone et va même jusqu'à être présentée comme la solution à la crise linguistique que traversait le Québec il y a 40 ans.

Les auteurs abordent aussi à maintes reprises l'importance qu'a eue la loi 101 sur le bilinguisme des Anglo-Québécois et sur la francisation des allophones:

(6) Le contexte a énormément changé depuis 1977. La proportion d’anglophones connaissant le français est passée de $37 \%$ à $70 \%$. La proportion d'allophones connaissant le français a bondi de $47 \%$ à $77 \%$. Et surtout, le taux de fréquentation de l'école française par les jeunes allophones est passé de $15 \%$ à 89 \%. (Cardinal, La Presse, 26 août 2017) 
(7) Bill 101 also pushed once unilingual anglos to send their kids to French schools, or immersion - where they became comfortably bilingue. (Freed, The Gazette, 27 mai 2017)

Ces arguments, souvent chiffrés, servent à montrer le renversement qui s'est opéré au lendemain de l'adoption de la loi 101. Rappelons que jusqu'aux années 1960, l'instruction publique était divisée au Québec : les écoles catholiques de langue française, d'un côté, et les écoles protestantes de langue anglaise, de l'autre. Les premières n'acceptaient en leurs rangs que les enfants de confession catholique, alors que les secondes accueillaient tous les autres, qu'ils soient ou non protestants. Cette répartition des élèves québécois sur le critère de la religion favorisait l'intégration des nouveaux arrivants, souvent de confession autre que catholique, à l'école anglaise et, par le fait même, à la communauté anglophone (Deshaies, Ouellon et Rocheleau, 1998). Par ailleurs, l'anglais étant considéré à l'époque comme la langue de promotion sociale, plusieurs parents souhaitaient que leurs enfants fréquentent l'école anglaise afin de leur assurer un avenir jugé meilleur ${ }^{8}$. À cela s'ajoute, comme nous l'avons mentionné, que jusqu'à l'adoption de la loi 101, c'était plus ou moins le libre-choix en matière de langue d'enseignement qui prévalait au Québec', avec pour conséquence que l'école de langue française était délaissée par plusieurs au profit de l'école de langue anglaise. Aujourd'hui, le renversement de la tendance qu'avaient les enfants d'immigrants à fréquenter presque exclusivement l'école anglaise et la francisation massive des anglophones et des allophones sont considérés par plusieurs, ce dont témoignent les exemples 6 et 7, comme l'une des plus grandes réussites de la loi 101 (Levine, 1997 ; Saint-Laurent, 2008 ; Oakes et Warren, 2009).

Le fait que de tels aspects positifs associés à la loi 101 soient nombreux dans le corpus et qu'ils soient présents dans la quasi-totalité des articles peut paraître signe de consensus, voire de cohésion sociale, qui, après des décennies de conflit linguistique, mérite d'être applaudi. Or, ces éléments en faveur de la loi 101 se trouvent majoritairement, dans les structures concessives qui les contiennent, en position d'énoncé concédé. Autrement dit, les auteurs concèdent certes des points positifs à la loi 101, mais ils contre-argumentent au moyen d'aspects négatifs qui entraînent à leur tour une conclusion où est jeté un regard critique sur la loi 101, regard qui est toutefois motivé par de multiples facteurs.

8. Cet argument revient aussi, quoique sous une forme légèrement modifiée, dans le débat actuel sur la loi 101. Il en sera question ultérieurement.

9. Si, officiellement, le libre-choix en matière de langue d'enseignement a été restreint après l'adoption de la loi 22 en 1974, cette restriction a été minimale : l'accès à l'école de langue anglaise était permis dès lors que les écoliers démontraient, à la suite de tests de langue (aisément contournables), des connaissances suffisantes de l'anglais. Corbeil (2007: 180) mentionne qu'en 1976, seules $15 \%$ des demandes d'admissibilité à l'école anglaise ont été refusées. 


\subsection{Regards négatifs sur la loi 101}

C'est en comparant les quatre journaux à l'étude que l'on peut observer la plus grande différence entre les regards négatifs posés sur la loi 101.

Ce sont uniquement les journaux francophones - et encore, seuls Le Devoir et Le Journal de Montréal - qui présentent le discours de personnes qui jugent que malgré les gains permis par la loi 101, celle-ci n'est pas appliquée de façon suffisamment rigoureuse ou, élément encore plus souvent mis de l'avant, qu'elle ne va pas assez loin. Les auteurs, même après avoir soulevé certains des points positifs présentés précédemment, en arrivent alors à la conclusion que la loi 101 ne constitue pas une pleine réussite et qu'elle devrait être sinon repensée, du moins resserrée. C'est ce qui transparaît des deux exemples suivants:

(8) La loi 101 voulait, très simplement, faire du français la langue «normale et habituelle » du travail, de l'enseignement, de l'État et du commerce. C'était une loi de « décolonisation» politique et psychologique des Québécois. Si on compare la scolarisation des enfants d'immigrants ou l'affichage commercial d'aujourd'hui à ce qu'ils étaient il y a 40 ans, les progrès ont été spectaculaires. Le problème est que les reculs sont tout aussi évidents depuis 20 ans. (Facal, Le Journal de Montréal, 26 août 2017)

(9) L'adoption de la Charte de la langue française est incontestablement un exploit. Elle a permis des avancées considérables au chapitre de la cohésion sociale, de l'intégration des immigrants par l'école et le marché du travail. Mais dès le début des années 1980, elle a subi les assauts des tribunaux, qui l'ont interprétée de façon restrictive et qui ont limité sa portée. Sur le plan psychologique, elle a ravivé la fierté des francophones tout en leur conférant un sentiment de fausse sécurité. Les avancées de la loi 101 ont été tenues pour acquises et les générations qui ont suivi ont failli à leur devoir de transmettre leur amour de la langue et de la culture aux générations futures. Résultat: le français est en recul comme langue maternelle et comme langue d'usage et la culture d'expression française est noyée dans le magma de l'uniformisation anglo-saxonne. L'enseignement du français souffre de lacunes graves et les cours de francisation des immigrants sont inadéquats et insuffisants. Des universités francophones offrent des programmes de formation en anglais et le français recule comme langue de travail. Ce ne sont là que quelques constats qui sautent aux yeux. Pourtant, le renforcement de la langue nationale est loin d'être une priorité du gouvernement. D'où l'importance d'ouvrir un chantier de la langue française afin d'apporter les correctifs qui s'imposent avant qu'il ne soit trop tard. (Houda-Pepin, Le Devoir, 26 août 2017) 
Dans ces extraits, les auteurs concèdent que la loi 101 a permis de nombreux progrès, notamment en matière d'enseignement, d'affichage ou de travail, et jugent très favorablement sa mise en œuvre dans les années 1970, allant même jusqu'à en parler en termes d'exploit. L'accent est toutefois mis sur des éléments présentés comme alarmants : les régressions en matière d'acquis linguistiques (exemple 8) et une série de critiques allant du recul du français comme langue maternelle et comme langue de travail aux lacunes dans le domaine de l'enseignement et à l'inaction du gouvernement en ce qui a trait à la question linguistique (exemple 9).

Ces exemples illustrent bien le concept de majorité fragile proposé par Marie McAndrew (2010). II s'agit, selon l'auteure, de communautés qui proviennent de sociétés divisées à dominance ambiguë, ce qui s'applique au Québec ${ }^{10}$, où la communauté francophone peut être envisagée à la fois comme une minorité ou une majorité selon le cadre de référence choisi : majoritaire au plan provincial et minoritaire par rapport à l'État canadien. Si les Québécois francophones constituent une communauté linguistique majoritaire à bien des égards (démographique, certes, mais aussi politique, culturelle, etc.) depuis l'adoption de la loi 101, leur existence est néanmoins parfois ressentie comme fragile par certains membres du groupe. Selon McAndrew, la communauté francophone du Québec est en processus de majorisation, ce dont témoignent aussi les extraits précédents par la concession des avancées réalisées après la promulgation de la loi 101, mais la fragilité qui l'accompagne, qu'illustrent les contre-arguments, reflète ce processus non achevé. Cette impression de fragilité transparaît tout particulièrement du dernier extrait, qui présente une conclusion explicite sous forme d'injonction: il faut intervenir avant qu'il ne soit trop tard. Le bilan anniversaire sert ici non seulement à effectuer un retour sur 40 ans d'application de la Charte de la langue française, mais aussi à appeler à la mobilisation et à l'action collective, notamment face à un gouvernement jugé peu préoccupé - et peu intéressé - par les questions linguistiques.

Du côté de la Gazette, et dans une moindre mesure de La Presse ${ }^{11}$, au contraire, la principale critique est à l'effet que la loi 101 va trop loin ou que certaines de ses dispositions, si elles étaient nécessaires dans le contexte des années 1970, ne le sont plus aujourd'hui. Le statut du français au Québec est alors considéré comme acquis - ce qui tranche nettement avec les discours publiés dans Le Devoir et dans Le Journal de Montréal - et les mesures protectionnistes à son égard sont jugées superflues, voire entraînant des conséquences négatives. Il est tout particulièrement question de l'érosion de la communauté anglophone et de ses institutions :

(10) But those efforts can only accomplish so much, and it's members of the English-speaking minority who are worried about survival now. Historic English communities in the regions are in decline. While Montreal's remains vibrant, there has been an erosion of our institutions. Clearly, Quebec anglophones have little reason to celebrate Bill 101 [...] (Équipe éditoriale, The Gazette, 25 août 2017)

10. L'auteure présente aussi l'exemple de la Flandre, de la Catalogne et de l'Irlande du Nord.

11. Rappelons que seuls cinq textes d'opinion à propos du 40e anniversaire de la loi 101 ont été relevés dans ce journal. 
Or, s'il est vrai que les rapports de force entre francophones et anglophones ont été modifiés depuis 1977, entraînant inévitablement des restructurations au sein de la communauté anglophone, il n'en demeure pas moins que la minorité anglophone jouit d'un statut enviable, notamment lorsqu'on la compare avec les minorités francophones canadiennes hors Québec (Bourhis et Landry, 2002). Malgré tout, ces transformations inquiètent, comme l'illustrent autant l'exemple précédent que de nombreux travaux portant sur la communauté anglo-québécoise (par exemple Caldwell, 2002 et Lamarre, 2007), ce qui amène certaines personnes à envisager la loi 101 avec méfiance. Cette crainte de l'érosion des institutions anglophones - et tout particulièrement du réseau scolaire primaire et secondaire ${ }^{12}$ - constitue le principal argument sur lequel se fondent les auteurs qui jugent que la loi 101 va trop loin.

Y est aussi souvent associée la croyance selon laquelle l'obligation de fréquenter une école de langue française pour la majorité des Québécois contribue non seulement à affaiblir les commissions scolaires anglophones, mais instaure également un bilinguisme inégal dont les francophones feraient les frais :

(11) The irony is that the young Quebec anglophone today is bilingual, and fully mobile, while the young francophone is unilingual and ghettoized. (Da Shiparo, The Gazette, 17 août 2017)

Cet argument n'est pas sans rappeler un des éléments marquants des conflits linguistiques des années 1960-1970 autour de la langue d'enseignement où, rappelons-le, l'anglais était considéré comme permettant d'ouvrir beaucoup plus de portes que le français. Il s'agit dans une certaine mesure de la réactualisation de cet argument, mais sous l'angle du bilinguisme et de la mobilité. Ces derniers, présentés comme nouveaux gages de réussite (Duchêne et Heller, 2012), seraient inaccessibles, selon certains auteurs du corpus, à ceux qui fréquentent l'école de langue française (à ce propos, voir aussi Vincent, 2010). Cela est d'autant plus mis de l'avant que les résultats du dernier recensement de Statistique Canada (2017) montrent que les Québécois anglophones et allophones sont effectivement, en général, plus bilingues (français-anglais) que les francophones. II n'en demeure pas moins que le Québec est la province où se trouvent le plus grand nombre de personnes bilingues (44,5\% contre 17,9\% pour l'ensemble du Canada) et que le taux de bilinguisme des Québécois francophones, d'environ $40 \%$, est tout de même considérable. La population francophone ne correspond donc pas exactement au portrait qui en est dressé dans l'exemple 11. Dans un autre ordre d'idées, cet argument laisse entrevoir des similitudes avec le discours qui prédominait avant l'adoption de la loi 101 et qui continue largement à circuler à l'échelle mondiale à l'heure actuelle: sous le couvert du bilinguisme, il est vrai, l'anglais est présenté comme symbole de réussite. Certes, les raisons diffèrent par rapport aux années 1960-1970 - de langue du groupe dominant, on passe à l'anglais comme langue hégémonique qui traverse les frontières, comme langue de la mondialisa-

12. Pour mémoire, les restrictions en matière de langue d'enseignement ne s'étendent pas au système d'éducation postsecondaire. Le réseau collégial et universitaire anglophone, dont la fréquentation demeure stable ou augmente légèrement (Olivier, 2017a, 2017b), ne fait pas l'objet d'inquiétude dans le discours de ces auteurs, contrairement à ce que l'on observe dans Le Devoir et dans Le Journal de Montréal. 
tion (Dewey, 2007) - mais il n'en demeure pas moins que la loi 101 est perçue par plusieurs comme limitant les possibilités, pour les Québécois francophones, de maîtriser cette langue et de profiter des avancements qu'elle est considérée permettre.

La communauté linguistique au cour des préoccupations soulevées dans les articles participe également à distinguer les journaux selon les jugements négatifs portés sur la loi 101. Au Devoir, à La Presse et au Journal de Montréal, il est question de la communauté francophone, sans surprise, mais aussi beaucoup des allophones. Ces derniers sont tantôt pointés du doigt lorsqu'il est question de ce qui est présenté comme le recul du français au Québec, tantôt jugés victimes du groupe majoritaire qui les exclut:

(12) Ce n'est pas sans raison qu'on a vu dans la loi 101 l'expression la plus ambitieuse de notre volonté de conserver et d'affirmer notre identité collective en Amérique. Mais que reste-t-il de la loi 101 ? En 40 ans, nous sommes passés du rêve d'un Québec français au Québec bilingue. On maquille cette régression en adaptation à la mondialisation. Dans les faits, le français perd du terrain. On le voit sur le marché du travail. On constate aussi qu'il est bien moins attrayant qu'on ne le souhaiterait pour un trop grand nombre d'immigrés, qui passent massivement dans le système scolaire anglophone lorsqu'ils arrivent au cégep, c'est-à-dire dès qu'ils en ont l'occasion. (Bock-Côté, Le Journal de Montréal, 26 août 2017)

(13) Il y a certes des secteurs qu'il faut continuer de surveiller avec vigilance, comme le milieu de travail et l'affichage commercial, mais la priorité, aujourd'hui, n'est pas le combat contre l'anglais : c'est l'utilisation du français par les immigrants, de plus en plus nombreux. Des immigrants qu'on francise grâce à la loi 101... mais qu'on abandonne ensuite en négligeant leur intégration sociale et économique. (Cardinal, La Presse, 26 août 2017)

À la Gazette, au contraire, ce sont les effets jugés néfastes de la loi 101 sur la communauté anglophone qui sont le plus mis de l'avant. Peu d'articles mettent l'accent sur les francophones et les allophones. On va même jusqu'à parler du 26 août 1977 comme d'une triste journée pour la communauté anglophone dans l'éditorial du journal. S'il n'est pas étonnant que chaque journal s'adresse à un lectorat différent, il est intéressant de noter la division linguistique qui s'opère : la communauté anglophone fait l'objet de très peu d'articles au Devoir, à La Presse et au Journal de Montréal, alors que les communautés francophone et allophone, quant à elles, sont presque absentes des discussions de The Gazette.

Enfin, les trois journaux de langue française, et à plus forte raison Le Journal de Montréal, remettent à l'avant-plan des discussions le débat sur la qualité de la langue au Québec. Plusieurs auteurs estiment qu'une des principales ratées de la loi 101 est de ne pas avoir insufflé aux francophones la fierté nécessaire pour qu'ils « respectent » (l'expression est souvent employée) la langue française. Pour ces auteurs, le respect de la langue est très nettement associé à l'absence d'écart à la norme 
linguistique, voire à un alignement sur la variété hexagonale, éléments caractéristiques du discours puriste (Paveau et Rosier, 2008) :

(14) Nous parlons encore joual, mais son évolution comprend de nouveaux ajouts, dont des mots anglais à la mode ou de gros mots anglo-saxons, le résultat en étant un mélange désarçonnant. On parle comme on tweete et vice-versa. Même les universitaires, les journalistes et les artistes, défenseurs officiels et traditionnels de la loi 101, se sont relâchés au fil des ans. La détérioration de la langue parlée et écrite s'est vécue dans une espèce de démocratisation pervertie. [...] L'ennemi n'est plus celui que l’on croit. (Bombardier, Le Journal de Montréal, 26 août 2017)

Dans cet extrait, l'auteure juge que si même les « défenseurs officiels et traditionnels de la loi 101 » se permettent un tel écart, caractérisé selon elle par le recours au joual - appellation fourre-tout servant à dévaluer le français en usage au Québec - et par la présence d'emprunts à l'anglais, c'est signe que l'avenir de la langue française est loin d'être assuré au Québec. La démocratisation dite pervertie de la langue, associée à sa détérioration, témoignerait sinon du rejet du français de la part des Québécois francophones, du moins de leur désintérêt à son égard. Ce discours alarmiste, comme il en circule depuis des décennies au Québec (Bouchard, 1998), est mis en avant dès lors qu'est abordée la question de la langue dans l'espace public. Or, on observe sinon une confusion, du moins un amalgame entre les visées de la loi 101, qui a pour but d'agir sur le statut de la langue française au Québec, et des considérations relatives à la qualité de la langue. La remise en discours de tels propos ne permet pas d'établir un véritable bilan des réussites et des échecs de la loi 101, mais participe plutôt à alimenter les discours dévalorisants à l'endroit du français québécois, lieu commun lorsqu'il est question de langue au Québec, et, de là, l'insécurité linguistique des Québécois.

\section{Conclusion}

Quarante ans après l'adoption de la Charte de la langue française, le débat sur le rôle du français au Québec et sur la pertinence de la législation linguistique en place est loin d'être terminé. L'analyse menée ici, qui a permis de mettre en lumière les différents regards posés sur la loi 101 après quatre décennies d'application, en témoigne. Malgré une reconnaissance du caractère essentiel de la loi en 1977 et bien que plusieurs la considèrent comme une des lois les plus importantes de l'histoire du Québec, si ce n'est la plus importante, les avis divergent quant à son application dans le contexte actuel. Les tensions entre les éléments positifs et négatifs associés à la loi sont nombreuses et leur mise en discours, dans les journaux à l'étude, est révélatrice des dissensions entourant la Charte de la langue française encore aujourd'hui. 
En nous penchant sur le fonctionnement discursif et sur les effets argumentatifs de la concession, nous avons pu montrer non seulement l'existence de ces dissensions, mais aussi, et surtout, la façon dont les points de vue antagonistes sont présentés et hiérarchisés les uns par rapport aux autres. Plus qu'un débat où des opinions tranchées s'opposent selon des camps bien établis - construits sur des critères linguistiques ou politiques, par exemple -, les discours médiatiques qui constituent le bilan anniversaire de la loi 101 mettent en évidence toute la complexité de la perception de la législation linguistique dans l'espace public québécois. Les auteurs des articles analysés sont unanimes - ou presque - quant à l'importance de la loi 101 et ils portent un regard appréciatif sur cette dernière, principalement en ce qui a trait à l'émancipation des francophones dans les années 1970 et à la francisation des anglophones et des allophones. Or, la portée de ces arguments en faveur de la loi 101 est rapidement restreinte. En effet, toujours placés en position d'énoncé concédé, ces points positifs sont renversés, ou du moins amoindris, par des contre-arguments qui mettent en lumière les aspects jugés négatifs de la loi 101, aspects toutefois fort éloignés au plan idéologique: pour certains, la loi 101 ne va pas assez loin, voire a déjà commencé à reculer et à mettre en péril la situation du français au Québec, alors que pour d'autres, au contraire, elle va trop loin et elle a un impact négatif sur le « vivre-ensemble » des communautés linguistiques. Ainsi se confrontent deux visions plutôt négatives de la loi 101, mais qui reposent sur des bases totalement différentes et qui appellent à des actions opposées : renforcer ou affaiblir, si ce n'est abroger, la loi 101.

S'il est attendu, dans le genre discursif qu'est le bilan anniversaire, qu'une forme d'alternance entre regards positifs et négatifs soit donnée à voir, il est néanmoins étonnant de constater comment, malgré leur polarisation, les points de vue sont hiérarchisés de façon identique. Ainsi, la conclusion générale de chaque article ou presque laisse entrevoir une forme de ressentiment envers la loi 101 - et parfois envers certaines communautés linguistiques - qui surpasse les avancées que cette dernière a permises. Quarante ans après son adoption, la loi 101 fait l'objet de critiques suffisamment nombreuses - et, surtout, provenant de toutes parts - pour que l'on s'interroge sur ce qui attend la législation linguistique québécoise dans les décennies à venir. 


\section{Références}

Amossy, Ruth (2014), Apologie de la polémique, Paris, Presses universitaires de France.

Amossy, Ruth (2010), L'argumentation dans le discours, 3e éd., Paris, Armand Colin. [1'e éd., 2000.]

Bernard, André (2008), « Les répercussions sociales et politiques de la Loi 101 », dans Michel Plourde et Pierre Georgeault (dir.), Le français au Québec : 400 ans d'histoire et de vie, 2 éd., Montréal, Fides, p. 360-368. [1 éd., 2000.]

Bouchard, Chantal (1998), La langue et le nombril : histoire d'une obsession québécoise, Montréal, Fides.

Bourhis, Richard et Rodrigue Landry (2002), « La loi 101 et l'aménagement du paysage linguistique au Québec », Revue d'aménagement linguistique, numéro hors série (L'aménagement linguistique au Québec : 25 ans d'application de la Charte de la langue française), p. 107-131.

Caldwell, Gary (2002), « La Charte de la langue française vue par les anglophones », Revue d'aménagement linguistique, numéro hors série (L’aménagement linguistique au Québec : 25 ans d'application de la Charte de la langue française), p. 27-34.

Corbeil, Jean-Claude (2007), L'embarras des langues : origine, conception et évolution de la politique linguistique québécoise, Montréal, Québec Amérique.

Danon-Boileau, Laurent et Mary-Annick Morel (1992), «Opérations énonciatives et valeurs argumentatives : étude de trois marqueurs concessifs », Approches énonciatives de l'énoncé complexe, Louvain, Peeters, p. 7-19.

Deshaies, Denise, Conrad Ouellon et Claude Rocheleau (1998), «L'aménagement linguistique au Québec : historique et perspectives d'avenir », dans Alain Bélanger, Nubia Hanciau et Sylvie Dion (dir.), L’Amérique française : introduction à la culture québécoise, Rio Grande, Editora da furg, p. 89132.

Dewey, Martin (2007), «English as a lingua franca and globalization : an interconnected perspective », International Journal of Applied Linguistics, vol. 17, n³ 3, p. 332-354.

Dominicy, Marc et Madeleine Frédéric (dir.) (2001), La mise en scène des valeurs : la rhétorique de l'éloge et du blâme, Lausanne, Paris, Delachaux et Niestlé.

Duchêne, Alexandre et Monica Heller (dir.) (2012), Language in late capitalism : pride and profit, Londres, Routledge.

Fowler, Roger (1991), Language in the News: Discourse and Ideology in the Press, Londres, Routledge.

Gouvernement du Québec (1977), La politique québécoise de la langue française présentée à l'Assemblée nationale et au peuple du Québec, Québec, Éditeur officiel du Québec.

Krieg-Planque, Alice (2010), « La formule "développement durable” : un opérateur de neutralisation de la conflictualité », Langage et société, n 134, p. 5-29. 
Lamarre, Patricia (2007), «Anglo-Quebec today : looking at community and schooling issues », International Journal of the Sociology of Language, vol. 185, p. 109-132.

Levine, Marc (2002), «La question "démolinguistique”, un quart de siècle après la Charte de la langue française ", Revue d'aménagement linguistique, numéro hors série (L’aménagement linguistique au Québec : 25 ans d'application de la Charte de la langue française), p. 165-181.

Levine, Marc (1997), La reconquête de Montréal, Montréal, VLB éditeur.

McAndrew Marie (2010), Les majorités fragiles et l'éducation: Belgique, Catalogne, Irlande du Nord, Québec, Montréal, Les Presses de l'Université de Montréal.

McAndrew, Marie (2002), « La Loi 101 en milieu scolaire : impacts et résultats », Revue d'aménagement linguistique, numéro hors série (L'aménagement linguistique au Québec : 25 ans d'application de la Charte de la langue française), p. 69-82.

Morel, Mary-Annick (1996), La concession en français, Paris, Ophrys.

Oakes, Leigh (2004), «Le français comme "langue publique commune" au Québec », Globe. Revue internationale d'études québécoises, vol. 7, n², p. 155-176.

Oakes, Leigh et Jane Warren (2009), Langue, citoyenneté et identité au Québec, Québec, Presses de l'Université Laval.

Olivier, Charles-Étienne (2017a), Langue et éducation au Québec, vol. 2 (Enseignement collégial), Québec, Office québécois de la langue française.

Olivier, Charles-Étienne (2017b), Langue et éducation au Québec, vol. 3 (Enseignement universitaire), Québec, Office québécois de la langue française.

Paveau, Marie-Anne et Laurence Rosier (2008), La langue française : passions et polémiques, Paris, Vuibert.

Perelman, Chaïm et Lucie Olbrechts-Tyteca (1988), Traité de l'argumentation : la nouvelle rhétorique, $5^{e}$ éd., Bruxelles, Éditions de l'Institut de Sociologie de l'Université Libre de Bruxelles. [1re éd., 1958.]

Pernot, Laurent (1993), La rhétorique de l'éloge dans le monde gréco-romain, Paris, Institut d'Études augustiniennes.

Rocher, Guy (2008), « La politique et la loi linguistiques du Québec en 1977 », dans Michel Plourde et Pierre Georgeault (dir.), Le français au Québec : 400 ans d'histoire et de vie, $2^{2}$ éd., Montréal, Fides, p. 339-353. [1'éd., 2000.]

Saint-Laurent, Nathalie (2008), Le français et les jeunes, Québec, Conseil supérieur de la langue française.

Sarrazin, Marie-Claude (2002), "L'évolution de la situation linguistique au Québec : le regard porté par une enfant de la Loi 101 », Revue d'aménagement linguistique, numéro hors série (L'aménagement linguistique au Québec: 25 ans d'application de la Charte de la langue française), p. 133-145. 
Statistique Canada (2017), Un nouveau sommet pour le bilinguisme français-anglais, disponible sur http://www12.statcan.gc.ca/census-recensement/2016/as-sa/98-200-x/2016009/98-200x2016009-fra.cfm. [Page consultée le 12 janvier 2018.]

Vincent, Diane (2010), « Langues nationales, variétés régionales et immigration : les défis de l'école en français au Québec », dans Benoît Falaize, Charles Heimberg et Olivier Loubes (dir.), L'école et la nation, Lyon, ENS Éditions, p. 235-246.

Vincent, Diane et Troy Heisler (1999), «L'anticipation d’objections : prolepse, concession et réfutation dans la langue spontanée », Revue québécoise de linguistique, vol. 27, n 1, p. 15-31.

Woehrling, José (2005), «L'évolution du cadre juridique et conceptuel de la législation linguistique du Québec », dans Alexandre Stefanescu et Pierre Georgeault (dir.), Le français au Québec : les nouveaux défis, Montréal, Fides, p. 253-356. 